\title{
Feasibility and effects of an exercise- based intervention in prison inmates with psychiatric disorders: the PsychiActive project randomized controlled trial
}

Clinical Rehabilitation I-I I

(C) The Author(s) 2019

Article reuse guidelines:

sagepub.com/journals-permissions DOI: $10.1177 / 0269215519845133$ journals.sagepub.com/home/cre @SAGE

\author{
Javier Bueno-Antequera ${ }^{1,2}[$, Miguel Ángel Oviedo- \\ Caro $^{1,2}$ and Diego Munguía-Izquierdo',2,3
}

\begin{abstract}
Objective: To evaluate the feasibility and effects of a 12-week intervention combining aerobic and strength exercises in prison inmates with psychiatric disorders.

Design: Two parallel-group, randomized controlled trials.

Setting: A psychiatric prison hospital.

Subjects: Forty-one men prison inmates (mean age $\pm S D=38.2 \pm 9.2$ years, mean prison duration \pm $S D=2.6 \pm 2.5$ years) with psychiatric disorders (primarily personality disorder, $n=27$; mean illness duration $\pm S D=12.0 \pm 10.5$ years).

Interventions: Participants were randomly allocated to intervention group consisted of exercise plus usual care $(n=21)$ or control group which received usual care $(n=20)$ for 12 weeks. The exercise programme included three weekly sessions of group-based moderate-to-high intensity combined exercises designed and supervised by exercise professionals.

Main measures: Fitness and anthropometric measures were assessed using field-based tests (6-minute walk, Incremental Shuttle Walk, Arm-Curl, and Chair-Stand), handgrip dynamometry, bioelectrical impedance, and waist and hip circumferences.

Results: There were no adverse events, and 10 intervention participants withdrew. The remaining II participants attended a mean of 28 sessions, of which nine met the compliance criteria. Betweengroup change differences substantially favoured the compliance intervention group for the 6-minute walk (+21.2\%), Incremental Shuttle Walk (+33.9\%), Arm-Curl (+ 13.8\%), waist (-3.5\%), waist/height ${ }^{0.5}(-1.7 \%)$ $(-2.7 \%)$, waist/hip $(-3.4 \%)$, and Body Shape Index (-3.3\%) (-3.5\%). Additional analysis showed beneficial effects of exercise participation on handgrip strength.

Conclusion: The intervention was safe, had a high dropout rate, and seemed to be effective for improving fitness and anthropometric measures in men prison inmates with psychiatric disorders who attended and participated in the exercise sessions.
\end{abstract}

'Physical Performance \& Sports Research Center, Department of Sports and Computer Science, Section of Physical

Education and Sports, Faculty of Sports Sciences, Universidad Pablo de Olavide, Seville, Spain

${ }^{2}$ Research Group in Development Movimiento Humano, Universidad de Zaragoza, Zaragoza, Spain

${ }^{3}$ Biomedical Research Networking Center on Frailty and

Healthy Aging, Madrid, Spain

\section{Corresponding author:}

Javier Bueno-Antequera, Department of Sports and Computer Science, Faculty of Sports Sciences, Universidad Pablo de Olavide, Carretera Utrera, Km. I, s/n, Seville 4I0I3, Spain.

Email: jbueant@upo.es 


\section{Keywords}

Mental disorders, exercise, physical fitness, anthropometry, prisons

Received: 23 August 2018; accepted: 29 March 2019

\section{Introduction}

Psychiatric disorders is a leading cause of cardiovascular disease ${ }^{1}$ and premature all-cause death ${ }^{2}$ worldwide. In 2010, the global economic burden of psychiatric disorder was comparable to that of cardiovascular diseases and higher than that of cancer, chronic respiratory diseases, and diabetes, and is expected to double by $2030 .^{3}$ Therefore, improving treatment for this clinical population is a global health priority.

A plethora of evidence ${ }^{4,5}$ has consistently demonstrated that exercise-based interventions are a feasible, acceptable, and effective manner to improve both mental and physical health in this population. However, people with psychiatric disorders are $50 \%$ more likely than the general population not to meet physical activity guidelines. ${ }^{6}$ Given that prison inmates also do not undertake regular physical activities, ${ }^{7}$ it is possible that the combination of being a prison inmate and having a psychiatric disorder may exacerbate the tendency towards physical inactivity. There are very few peer-review studies on increasing exercise in the prison population, ${ }^{8-10}$ and none investigating exclusively prisoners with psychiatric disorders. Understanding the feasibility and effects of exercise-based interventions on the health of prison inmates with psychiatric disorders is both a challenge and an opportunity for the public health and scientific community.

An essential aspect of understanding the effects of interventions on health is the use of adequate measures. Physical fitness ${ }^{11,12}$ and anthropometric $^{13}$ measures have extensively shown to be strong independent predictors of all-cause and specificcause morbidity and mortality. The aim of the current study was to evaluate the feasibility and effects of an exercise-based intervention programme on the fitness and anthropometric measures of prison inmates with psychiatric disorders. We hypothesized that the exercise programme would improve the aforementioned variables.

\section{Material and methods}

The study was part of the PsychiActive project and followed CONSORT (Consolidated Standards of Reporting Trials) ${ }^{14}$ and CERT ${ }^{15}$ (Consensus on Exercise Reporting Template) guidance. The protocol was registered at ClinicalTrials.gov (Identifier: NCT03352544) and was approved by the Hospitales Universitarios Vírgen Macarena and Virgen del Rocío Ethics Committee (1674-N-17) and the Spanish Ministry of Interior. All participants gave their informed written consent prior to enrolling in the study and after receiving information about the aims and protocol. There was no compensation for participation.

The study was conducted in a psychiatric prison hospital (Hospital Psiquiátrico Penitenciario de Sevilla, Spain). Subjects were recruited over a 4-week period by clinical staff members, comprising nurses, doctors, psychologists, and social workers. The medical chief selected those who met the requirements to participate. Subjects were included if they had a psychiatric disorder diagnosed by experienced psychiatrists, were aged between 18 and 65 years, and were stabilized on antipsychotic medication during the last month. Subjects with clinical instability, substance abuse, or any other condition contraindicating participation were excluded.

Baseline assessment was performed before the intervention (in April 2015) and included demographic, clinical, fitness, and anthropometric data measures. Follow-up assessment was performed after 12 weeks of intervention (in July 2015) and included fitness and anthropometric measures. Each assessment period lasted a week. After baseline measurements were taken, participants were allocated to the control (usual care) or intervention (exercise + usual care) group, according to a balanced block randomization process using SPSS ( $50 \% / 50 \%$ of all the cases) conducted by a researcher (D.M.-I.) not involved in the assessment. The intervention started 1 week after randomization. Baseline 
and follow-up assessments, design, implementation, and supervision (face-to-face) of the intervention throughout the study period were conducted by two exercise physiologists (J.B.-A. and M.A.O.-C.), with more than 3 years of experience researching the subject of exercise in people with psychiatric disorders.

\section{Measures}

Feasibility was measured as follows:

- Recruitment rate: number of randomized participants divided by number of enrolled subjects.

- Attendance: number of exercise sessions the participant completed prescribed exercises.

- Persistence: number of weeks the participant attended at least one exercise session.

- Dropout: number of randomized participants who did not complete their treatment.

- Reasons for non-attendance and dropout.

Cardiorespiratory fitness was assessed with the distance walked (without running or jogging) in the 6-minute walk test (to the nearest $0.1 \mathrm{~m}$ ) and in the Incremental Shuttle Walk Test (to the nearest $10 \mathrm{~m}$ ) according to Rikli and Jones ${ }^{16}$ and Singh et al., ${ }^{17}$ respectively. To ensure maximal effort and avoid ceiling effect in the Incremental Shuttle Walk Test, the modified version without limiting by levels of velocities was used.

Handgrip strength was assessed to the nearest $0.1 \mathrm{~kg}$ with a hand dynamometer (TKK 5401 Grip-D, Takei, Tokyo, Japan). Participants in erect stance and with the arm in complete extension were instructed to squeeze the handle as fast and as hard as possible for 5 seconds. The test was performed twice (alternately with both hands) with a 1-minute rest between trials, and the maximum value of the four attempts was used. To account for individual differences in body mass, we have used relative grip strength (i.e. handgrip strength/body mass, both in $\mathrm{kg}$ ) for the analysis. ${ }^{18}$

Upper- and lower-body strength were measured using the 30-second Arm-Curl test and the 30-second Chair-Stand test, respectively, as explained elsewhere. ${ }^{16}$ For the Arm-Curl test, the best score of the two attempts (one for each arm and with a 1-minute rest between trials) was used. Concerning the Chair-Stand test, only one attempt was performed.

For each fitness test, except for handgrip test, multimedia explanations are available on the following link: https://upotv.upo.es/series/58da216a2 $38583 \mathrm{e} 0478 \mathrm{~b} 48 \mathrm{f0}$. For each test, the same instructor explained the protocol, gave a demonstration prior to start, supervised, verbally encouraged participants (using standardized encouragements for the 6-minute walk test ${ }^{16}$ ), and recorded the results.

Anthropometric measures were collected in the morning after an overnight fast. Height was measured to the nearest $0.1 \mathrm{~cm}$ with a stadiometer. Weight, fat mass, and fat-free mass were measured to the nearest $0.1 \mathrm{~kg}$ via bioelectrical impedance analysis (InBody-770, Biospace, Seoul, Korea). Waist circumference was assessed at the midpoint between the last rib and the iliac crest, and hip circumference at the level of the greater trochanter. Circumferences were measured twice to the nearest $0.1 \mathrm{~cm}$ using a measuring tape (Harpenden Anthropometric Tape; Holtain, Dyfed, UK), and mean values were used. Then, following anthropometric indices were calculated:

- Body mass index $=$ weigh $/$ height $^{2}$; weight in $\mathrm{kg}$ and height in $\mathrm{m}$.

- $\quad$ Fat mass index $=$ fat mass $/$ height $^{2}$; fat mass in $\mathrm{kg}$ and height in $\mathrm{m}$.

- Fat-free mass index = fat-free mass $/$ height $^{2}$; fat-free mass in $\mathrm{kg}$ and height in $\mathrm{m}$.

- Waist/height ${ }^{0.5}=$ waist circumference/ height $\mathrm{t}^{0.5}$; both in $\mathrm{m}$ and $\mathrm{cm}$.

- Waist/hip = waist circumference/hip circumference; both in $\mathrm{cm}$.

- A Body Shape Index = waist circumference/ body mass index $^{2 / 3} \times$ height $^{1 / 2}$; waist and height in $\mathrm{m}$ and body mass index in $\mathrm{kg} / \mathrm{m}^{2}$.

Age, diagnoses, illness and prison duration, and medication were obtained from the participants' medical records. Antipsychotic medication was converted into chlorpromazine equivalent dose. ${ }^{19}$ The Global Severity Index of the Brief Symptom 
Inventory- $18^{20}$ was used to assess psychopathological severity over the past week. Smoking was self-reported.

Intervention. The exercise programme lasted 12 weeks and included three weekly sessions (Monday, Wednesday, and Friday) of group-based aerobic and strength exercises, detailed in Supplemental Appendix 1, and accompanied by the participants' preferred choice of music. In each session, the two supervisors first explained the exercise to be performed during the tasks, continuously reinforced exercise techniques, giving positive feedback, and encouraging participants to do their best throughout the session, and commended participants for their efforts at the end. Exercise duration and intensity were monitored with the SenseWear Mini armband (BodyMedia Inc., Pittsburgh, PA, USA), which combines triaxial-accelerometry with measurements of heat production, to accurately estimate energy expenditure during aerobic, resistance, and combined exercise. ${ }^{21}$ All attendants wore the device on their left arm triceps muscle while training.

Usual care consisted of psychotherapy, pharmacological treatments, and group therapy (cognitive, educational, and creative/recreational activities such as painting and reading) facilitated by psychologists and social workers.

\section{Statistical analysis}

Analyses were performed on intention-to-treat and per-protocol bases. Intention-to-treat analyses included all randomized participants who provided baseline and follow-up data for every outcome measure. Per-protocol analyses included participants who attended at least $70 \%$ of the exercise sessions and the entire control group.

Between-group comparisons of baseline demographic and clinical characteristics were performed using unpaired $t$-tests (Student's for intention-totreat and Welch's for per-protocol), Mann-Whitney $U$-test, chi-square test, or Fisher exact test, according to the nature and distribution of variable. Between-group comparisons of the percent change in fitness and anthropometric measures were performed using unpaired $t$-tests (Student's or Welch's) and Mann-Whitney $U$-test. These tests were carried out using SPSS Statistics for Windows, version 22.0 (IBM Corp., Armonk, NY), and adjustments for multiple comparisons were made using the Bonferroni method by dividing the significance level of 0.050 by the number of comparisons. Data were also assessed for practical/clinical meaningfulness using an approach based on the magnitudes of change. Cohen's $d$ statistic determined the effect size of the standardized differences, and Hopkins' scale ${ }^{22}$ and a customized spreadsheet ${ }^{23}$ were used to determine the magnitude of the effect size.

\section{Results}

A participant flow diagram is shown in Figure 1, and baseline demographics data are presented in Table 1. A mean of $10.5 \pm 2.3$ participants (range, 6-16) from the intervention group attended each session, and duration of attendance was detailed in Table 2. Mean attendance and persistence for the 11 participants who completed the exercise programme were 28 sessions (range, 8-35), and 11 weeks (range, 7-12), respectively. Reasons for missed sessions were temporary exit from prison $(29 \%)$ (due to permission (50\%), participation in external activities organized by local mental health associations (38\%), and trial attendance (12\%)), conflicting schedules with training courses/prison work $(24 \%)$, mental ill health (18\%), physical ill health $(11 \%)$, unknown $(11 \%)$, and unspecified (7\%). No adverse events occurred during the study for either the intervention or control group.

Baseline and changes in fitness and anthropometric measures in the intention-to-treat and perprotocol analyses are shown in Supplemental Table S1 and Table 3, respectively. The control group exhibited worse baseline values for fitness and anthropometric measures compared to the intervention group. Between-group change differences substantially favoured the intervention group for cardiorespiratory fitness and several anthropometric in both analyses. In addition, the per-protocol analysis showed substantial benefits for the intervention group in upper-body strength. 


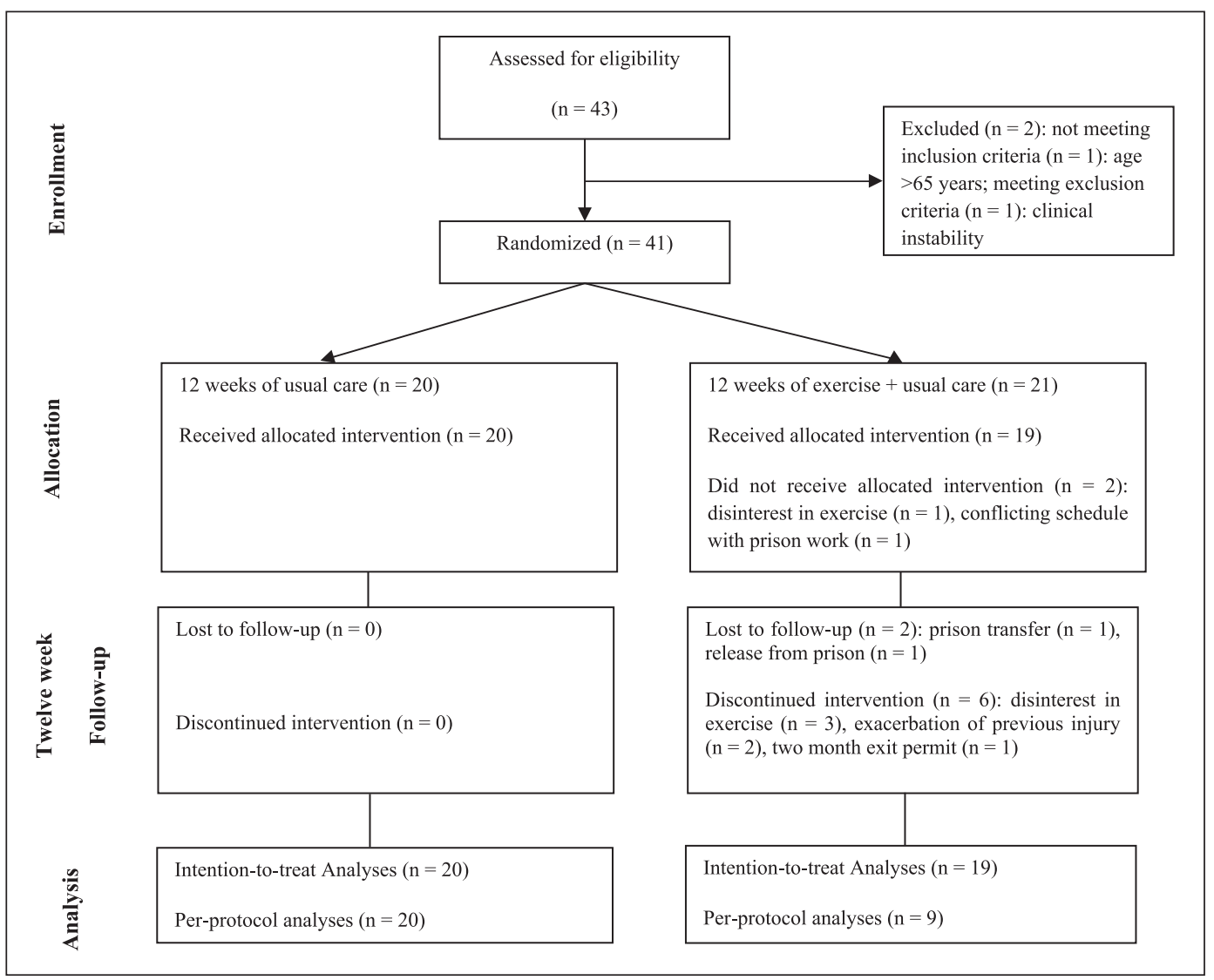

Figure I. Participants flow diagram.

Additional analysis showed that the number of participants with low handgrip strength $(<25$ th percentile of age- and gender-normative data from the National Health and Nutrition Examination Survey (NHANES) study ${ }^{18}$ ) increased in the control group (from 4 to 7 ) and decreased in the intervention group (from 2 to 0 , both of the compliance group).

\section{Discussion}

Two major findings of the present study can be highlighted. First, slightly more than half of individuals allocated to the intervention participated in the exercise programme. Second, the exercise programme produced substantial benefits in cardiorespiratory fitness, upper-body strength, and several anthropometric measures in those people who attended and participated in the exercise group sessions. Furthermore, additional analysis suggested beneficial effects of exercise participation in handgrip strength.

Our rates of attendance $(77 \%)$ and exercise dropout $(48 \%)$ were similar to those reported in previous studies of combined exercise in prison, which ranged from $57 \%$ to $75 \% 8,10,24$ and from $12 \%$ to $50 \%,{ }^{8-10,24}$ respectively. Interestingly, all of these studies were randomized controlled trials of groupbased interventions in men. In addition, the studies that administered combined exercise as the sole intervention component in adults with psychiatric disorders of the general community reported lower dropout (ranging from $0 \%$ to $36 \%)^{25-31}$ and similar attendance rates (ranging from $71 \%$ to $85 \%$ ), $25,26,28$ 
Table I. Characteristics of the participants.

\begin{tabular}{|c|c|c|c|c|c|}
\hline & All $(n=4 I)$ & $\begin{array}{l}\text { Control } \\
(n=20)\end{array}$ & $\begin{array}{l}\text { Intervention } \\
(n=2 I)\end{array}$ & $\begin{array}{l}\text { Compliance } \\
(n=9)\end{array}$ & $\begin{array}{l}\text { Non-compliance } \\
(n=12)\end{array}$ \\
\hline Age (years) & $38.2 \pm 9.2$ & $39.3 \pm 10.1$ & $37.1 \pm 8.3$ & $32.7 \pm 8.4$ & $40.4 \pm 6.7$ \\
\hline Illness duration (years) & $12.0 \pm 10.5$ & $11.7 \pm 11.3$ & $12.3 \pm 9.8$ & $6.8 \pm 4.1$ & $16.4 \pm 10.9$ \\
\hline Prison duration (years) & $2.6 \pm 2.5$ & $1.9 \pm 1.3$ & $3.3 \pm 3.2$ & $2.9 \pm 3.3$ & $3.7 \pm 3.3$ \\
\hline $\begin{array}{l}\text { Chlorpromazine } \\
\text { equivalent dose (mg/day) }\end{array}$ & $674.3 \pm 551.4$ & $789.7 \pm 661.9$ & $564.4 \pm 407.4$ & $569.3 \pm 390.3$ & $560.7 \pm 437.0$ \\
\hline $\begin{array}{l}\text { Psychopathological } \\
\text { severity }(0-72)^{\mathrm{a}, \mathrm{b}}\end{array}$ & $2.8 \pm 2.9$ & $3.4 \pm 3.5$ & $2.2 \pm 2.1$ & $2.1 \pm 2.2$ & $2.4 \pm 2.1$ \\
\hline $\begin{array}{l}\text { Smoking (current } \\
\text { smoker) }\end{array}$ & $32(78.0)$ & $18(90.0)$ & $14(66.7)$ & $8(88.9)$ & $6(50.0)$ \\
\hline \multicolumn{6}{|l|}{ Diagnoses } \\
\hline $\begin{array}{l}\text { Schizophrenia, } \\
\text { schizotypal and } \\
\text { delusional disorders }\end{array}$ & $2(4.9)$ & $2(10.0)$ & $0(0.0)$ & $0(0.0)$ & $0(0.0)$ \\
\hline $\begin{array}{l}\text { Mood [affective] } \\
\text { disorders }\end{array}$ & I (2.4) & $0(0.0)$ & I (4.8) & $0(0.0)$ & I (8.3) \\
\hline $\begin{array}{l}\text { Disorders of adult } \\
\text { personality and } \\
\text { behaviour }\end{array}$ & $27(65.9)$ & $13(65.0)$ & $14(66.7)$ & $6(66.7)$ & $8(66.7)$ \\
\hline $\begin{array}{l}\text { Neurotic, stress- } \\
\text { related, and } \\
\text { somatoform disorders }\end{array}$ & $5(12.2)$ & $2(10.0)$ & $3(14.3)$ & I (II.I) & $2(16.7)$ \\
\hline Mental retardation & $4(9.8)$ & I (5.0) & $3(14.3)$ & $2(22.2)$ & I (8.3) \\
\hline $\begin{array}{l}\text { Disorders due to } \\
\text { psychoactive substance } \\
\text { use }\end{array}$ & $2(4.9)$ & $2(10.0)$ & $0(0.0)$ & $0(0.0)$ & $0(0.0)$ \\
\hline
\end{tabular}

Values are the mean \pm standard deviation or $n(\%)$. Compliance included participants who attended at least $70 \%$ of the exercise sessions. Non-compliance included participants who dropped out $(n=10)$ and who attended less than $70 \%$ of the exercise sessions $(n=2)$.

aMissing data. Reasons: Incomplete questionnaire data for psychopathological severity (control $n=2$; intervention $n=2$, both of the non-compliance group).

bPsychopathological severity was assessed using the Brief Symptom Inventory-18, with higher scores indicating greater severity.

Table 2. Mean duration of attendance per session in minutes.

\begin{tabular}{|c|c|c|c|}
\hline & Mean & $S D$ & Range \\
\hline Total session & 49.6 & 12.9 & $24-66$ \\
\hline Total exercise ( $>$ I.5 METs) & 44.0 & 12.3 & $23-61$ \\
\hline $\begin{array}{l}\text { Light exercise ( }>1.5-3 \\
\text { METs) }\end{array}$ & 13.2 & 6.6 & $2-30$ \\
\hline $\begin{array}{l}\text { Moderate-to-vigorous } \\
\text { exercise (>3-9 METs) }\end{array}$ & 30.8 & 10.0 & $19-50$ \\
\hline $\begin{array}{l}\text { Very vigorous exercise ( }>9 \\
\text { METs) }\end{array}$ & 0.6 & 0.8 & $0-3$ \\
\hline
\end{tabular}

METs: metabolic equivalents.

Data are based on 342 valid SenseWear records. The three invalid SenseWear records were due to technical errors. Exercise duration and intensity (in METs) were derived using manufacturer-specific algorithms (SenseWear Professional software version 8.I; BodyMedia, Inc., Pittsburgh, PA, USA). 
Table 3. Baseline and between-group comparisons of the change from baseline to follow-up in fitness and anthropometric measures using per-protocol analyses.

\begin{tabular}{|c|c|c|c|c|c|c|}
\hline & \multirow[t]{2}{*}{$n$} & \multirow{2}{*}{$\begin{array}{l}\text { Baseline } \\
M \pm S D\end{array}$} & \multirow{2}{*}{$\begin{array}{l}\text { Change from } \\
\text { baseline (\%) } \\
M \pm S D\end{array}$} & \multirow{2}{*}{$\begin{array}{l}\begin{array}{l}\text { Between-group } \\
\text { difference }(\%)\end{array} \\
M(90 \% \mathrm{CL})\end{array}$} & \multicolumn{2}{|l|}{ Statistics } \\
\hline & & & & & ES $(90 \% C L)^{a}$ & $P$ \\
\hline \multicolumn{7}{|c|}{ 6-minute walk test $(\mathrm{m})^{\mathrm{b}}$} \\
\hline Control & 18 & $577.8 \pm 60.5$ & $-3.3 \pm 9.5$ & $21.2(13.3)$ & $1.23(0.77)^{* * * *}$ & 0.015 \\
\hline Intervention & 9 & $667.8 \pm 73.5$ & $17.9 \pm 20.7$ & & & \\
\hline \multicolumn{7}{|c|}{ Incremental Shuttle Walking Test $(m)^{b}$} \\
\hline Control & 18 & $484.4 \pm 156.7$ & $3.4 \pm 19.9$ & $33.9(24.3)$ & $1.35(0.65)^{* * * * *}$ & 0.005 \\
\hline Intervention & 9 & $697.8 \pm 201.1$ & $37.3 \pm 37.7$ & & & \\
\hline \multicolumn{7}{|c|}{ Relative handgrip strength ${ }^{b, c}$} \\
\hline Control & 20 & $0.533 \pm 0.089$ & $5.2 \pm 13.8$ & $-2.1(8.2)$ & $-0.16(0.64)$ & 0.662 \\
\hline Intervention & 9 & $0.596 \pm 0.109$ & $3.1 \pm 10.7$ & & & \\
\hline \multicolumn{7}{|c|}{ Arm-Curl test (repetitions) ${ }^{\mathrm{b}}$} \\
\hline Control & 19 & $27.6 \pm 6.4$ & $2.2 \pm 19.3$ & $13.8(\mid 1.9)$ & $0.76(0.65)^{* *}$ & 0.060 \\
\hline Intervention & 9 & $34.9 \pm 5.9$ & $16.0 \pm 15.8$ & & & \\
\hline \multicolumn{7}{|c|}{ Chair-Stand test (repetitions) ${ }^{\mathrm{b}}$} \\
\hline Control & 18 & $24.0 \pm 6.0$ & $4.3 \pm 21.6$ & $5.5(10.2)$ & $0.32(0.59)$ & 0.367 \\
\hline Intervention & 9 & $32.0 \pm 4.0$ & $9.8 \pm 9.3$ & & & \\
\hline \multicolumn{7}{|l|}{ Weight (kg) } \\
\hline Control & 20 & $89.6 \pm 18.4$ & $-0.3 \pm 6.6$ & $-1.4(3.4)$ & $-0.24(0.60)$ & 0.501 \\
\hline Intervention & 9 & $81.7 \pm 8.8$ & $-1.6 \pm 4.0$ & & & \\
\hline \multicolumn{7}{|c|}{ Body mass index $\left(\mathrm{kg} / \mathrm{m}^{2}\right)$} \\
\hline Control & 20 & $30.3 \pm 4.7$ & $-0.4 \pm 6.6$ & $-1.1(3.3)$ & $-0.19(0.60)$ & 0.595 \\
\hline Intervention & 9 & $27.5 \pm 3.7$ & $-1.5 \pm 3.8$ & & & \\
\hline \multicolumn{7}{|c|}{ Fat mass index $\left(\mathrm{kg} / \mathrm{m}^{2}\right)$} \\
\hline Control & 20 & $9.4 \pm 3.8$ & $4.8 \pm 19.8$ & $-4.9(21.0)$ & $-0.17(0.75)$ & 0.680 \\
\hline Intervention & 9 & $6.3 \pm 2.6$ & $-0.1 \pm 32.2$ & & & \\
\hline \multicolumn{7}{|c|}{ Fat-free mass index $\left(\mathrm{kg} / \mathrm{m}^{2}\right)$} \\
\hline Control & 20 & $20.9 \pm 2.0$ & $-1.8 \pm 4.5$ & $0.2(3.2)$ & $0.05(0.68)$ & 0.905 \\
\hline Intervention & 9 & $21.2 \pm 2.0$ & $-1.6 \pm 4.5$ & & & \\
\hline \multicolumn{7}{|l|}{ Waist $(\mathrm{cm})^{\mathrm{b}}$} \\
\hline Control & 20 & $103.9 \pm 13.0$ & $-0.7 \pm 6.5$ & $-3.5(3.7)$ & $-0.70(0.63)^{* *}$ & 0.099 \\
\hline Intervention & 9 & $93.8 \pm 8.5$ & $-4.1 \pm 4.8$ & & & \\
\hline \multicolumn{7}{|l|}{ Waist/height $0.5 \mathrm{~b}$} \\
\hline Control & 20 & $0.79 \pm 0.10$ & $-0.7 \pm 6.5$ & $-2.7(2.7)$ & $-0.73(0.63)^{* *}$ & 0.095 \\
\hline Intervention & 9 & $0.7 \mathrm{I} \pm 0.07$ & $-3.1 \pm 4.1$ & & & \\
\hline \multicolumn{7}{|l|}{ Waist/hip } \\
\hline Control & 20 & $1.012 \pm 0.063$ & $-3.4 \pm 4.4$ & $-3.4(2.6)$ & $-0.85(0.64)^{* *}$ & 0.033 \\
\hline Intervention & 9 & $0.961 \pm 0.034$ & $-6.8 \pm 3.4$ & & & \\
\hline \multicolumn{7}{|c|}{ A Body Shape Index } \\
\hline Control & 20 & $0.082 \pm 0.004$ & $-0.43 \pm 2.57$ & $-3.5(3.7)$ & $-0.74(0.74)^{* * *}$ & 0.039 \\
\hline Intervention & 9 & $0.079 \pm 0.003$ & $-4.12 \pm 4.80$ & & & \\
\hline
\end{tabular}

ES: effect size, CL: confidence limits.

Analyses included participants who attended at least $70 \%$ of the exercise sessions and control group participants who provided baseline and follow-up data for every outcome measure. Unpaired $t$-Welch's test was used for comparisons, except for Incremental Shuttle Walking Test and Waist. Mann-Whitney U-test was used in these cases. 
Table 3. (Continued)

aThreshold values for Cohen's ES were trivial (0.0-0.19), small (0.20-0.59), moderate (0.60-I.I9), large (I.20-I.99), and very large $(\geqslant 2.00)$. The numbers of asterisks $(*)$ indicate the likelihood for the between-groups differences to be substantial, with I symbol referring to possible difference, 2 to likely, 3 to very likely, and 4 to almost certain differences.

bMissing data. All were missing data from the follow-up except for one case in which a participant refused to perform the 6-minute walk test and Incremental Shuttle Walking Test both at baseline and at follow-up. In all cases, participants refused to perform the test except one in which a participant was injured and cannot perform the 6-minute walk, Incremental Shuttle Walking, and Chair-Stand tests at follow-up. cHandgrip strength/body mass, both in $\mathrm{kg}$. Significant when $P<0.004$ (i.e. $0.05 / 13$ comparisons $=0.004$ ).

with the exception of one study that had lower attendance $(37 \%)^{30}$ and another ${ }^{31}$ that had higher attendance $(97 \%)$. Considering the absence of adverse events, our combined exercise-intervention in men inmates with psychiatric disorders was safe and as feasible as other studies previously implemented in prisons and among people with psychiatric disorders in the general community.

The feasibility results may have been influenced by several aspects associated with reduced dropouts in people with psychiatric disorders, $5,32,33$ including (i) implementing a group-based intervention of (ii) moderate-to-high intensity exercise (iii) delivered by exercise professionals and (iv) supervised throughout the duration of intervention. Other factors, such as (i) the use of participant's preferred music ${ }^{34}$ and (ii) the variation of training sessions (comprising training methods, exercise selection, exercise equipment, and settings where exercises were performed), could have increased participation. On the contrary, the combined exercise training may have negatively influenced because it is associated with a greater dropout rate compared to aerobic or strength training alone in people with psychiatric disorders. ${ }^{32,33}$ However, all dropouts and missed training sessions were unrelated to the intervention. Despite this, the large dropout rate is worrying and represents a challenge for future exercise interventions in this context.

The improvement in fitness concurring with previous studies that compared combined exercise versus control in prison inmates ${ }^{8-10}$ and in people with psychiatric disorders in the general population, ${ }^{25-27}$ and may be partly due to some exercise programme characteristics. In particular, at least one weekly session included resistance circuit-based training using low-intensity exercises and lasted 24-60 minutes, which is an effective training method for the concurrent development of cardiorespiratory fitness and muscular strength in healthy adults. ${ }^{35}$ Furthermore, the implementation of an intervention programme with three weekly sessions and supervision by qualified exercise personnel may have also contributed to maximizing the cardiorespiratory fitness, which is in agreement with a recent meta-analysis of people with psychiatric disorders. ${ }^{36}$ Regarding muscular strength, the improvements may also be explained because a third of the sessions included resistance exercises of moderate intensity performed at maximal intended concentric velocity and moderate volume $(\sim 50 \%$ of the maximum number of possible repetitions), which results in higher enhancements in muscular strength than does moderate-slow resistance training performed at low intended concentric velocity ${ }^{37}$ and high volume. ${ }^{38}$

\section{Strengths and limitations}

One study strength was that the intervention was designed, implemented, and supervised by exercise physiologists. In addition, the duration and intensity of exercise were objectively recorded, while measurement errors were minimized by using the latest available model and algorithms of the activity monitor, and the limited amount of very highintensity exercise undertaken. ${ }^{21}$

The two major limitations were the loss of approximately half of participants from the intervention arm and the small sample of men, which reduce the power of the analyses and generalizability of the current findings. However, the rates of recruitment and completion of baseline and follow-up assessments were higher than $95 \%$, and the prison where the intervention took place is exclusively for men. 
The current study could help to raise health professionals' awareness of the importance of considering exercise as medicine in prison inmates with psychiatric disorders and may encourage the scientific community to research the feasibility and benefits of exercise programmes in prison environments. Implications derived from this study could be around how to engage this particular population in undertaking exercise. For this, there is extensive literature on how people with psychiatric disorders ${ }^{39}$ can be encouraged to participate in exercise that should be taken into account.

\section{Clinical messages}

- Approximately half of the eligible individuals allocated to the exercise intervention failed to participate.

- In those prison inmates prepared to undertake exercise, the provision of exercise within a group provided improvements in cardiorespiratory fitness, muscular strength, and anthropometric measures.

\section{Acknowledgements}

The authors gratefully acknowledge all participants for their collaboration. We also acknowledge the health clinic members involved in the recruitment for their effort and great enthusiasm. To access supplemental materials related to this work, contact the corresponding author.

\section{Author contributions}

J.B.-A. and D.M.-I. designed the study and wrote the protocol. All authors were responsible for the acquisition of the data. J.B.-A. and D.M.-I. performed the statistical analyses, and J.B.A. wrote the manuscript. All authors provided critical review of the manuscript and approved the final version.

\section{Declaration of conflicting interests}

The author(s) declared no potential conflicts of interest with respect to the research, authorship, and/or publication of this article.

\section{Funding}

The author(s) disclosed receipt of the following financial support for the research, authorship, and/or publication of this article: The work was funded by Research Group CTS-948, Universidad Pablo de Olavide, Andalusian Government; Universidad Pablo de Olavide, Spain, A2. Ayudas puente para la concurrencia al Plan Estatal de I + D (reference number PPI1802); European University of Madrid, Cátedra Real Madrid, Spain (reference number P2017/RM08); and Biomedical Research Networking Center on Frailty and Healthy Aging (CIBERFES) and FEDER funds from the European Union (reference number CB16/10/00477). JBA was supported by the Spanish Ministry of Education (reference number FPU13/05130). JBA and MAOC were supported by the Departamento de Innovación, Investigación y Universidad del Gobierno de Aragón y el Fondo Europeo de Desarrollo Regional - Programa Operativo FEDER Aragón 2014-2020, Construyendo Europa desde Aragón (reference numbers PUI/2018-337 (JBA) and PUI/2018-336 (MAOC)). The funders had no role in study design; collection, analysis, and interpretation of data; writing the report; and the decision to submit the report for publication.

\section{Supplemental material}

Supplemental material for this article is available online.

\section{ORCID iD}

Javier Bueno-Antequera (iD https://orcid.org/0000-0001 $-8063-3980$

\section{References}

1. Correll CU, Solmi M, Veronese N, et al. Prevalence, incidence and mortality from cardiovascular disease in patients with pooled and specific severe mental illness: a large-scale meta-analysis of 3,211,768 patients and 113,383,368 controls. World Psychiatry 2017; 16(2): 163-180.

2. Walker ER, McGee RE and Druss BG. Mortality in mental disorders and global disease burden implications: a systematic review and meta-analysis. JAMA Psychiat 2015; 72: 334-341.

3. Trautmann S, Rehm J and Wittchen HU. The economic costs of mental disorders: do our societies react appropriately to the burden of mental disorders? EMBO Rep 2016; 17(9): 1245-1249.

4. Rosenbaum S, Tiedemann A, Sherrington C, et al. Physical activity interventions for people with mental illness: a systematic review and meta-analysis. J Clin Psychiatry 2014; 75(9): 964-974. 
5. Firth J, Cotter J, Elliott R, et al. A systematic review and meta-analysis of exercise interventions in schizophrenia patients. Psychol Med 2015; 45(7): 1343-1361.

6. Vancampfort D, Firth J, Schuch FB, et al. Sedentary behavior and physical activity levels in people with schizophrenia, bipolar disorder and major depressive disorder: a global systematic review and meta-analysis. World Psychiatry 2017; 16(3): 308-315.

7. Elger BS. Prison life: television, sports, work, stress and insomnia in a remand prison. Int J Law Psychiatry 2009; 32(2): 74-83.

8. Battaglia C, di Cagno A, Fiorilli G, et al. Benefits of selected physical exercise programs in detention: a randomized controlled study. Int J Environ Res Public Health 2013; 10(11): 5683-5696.

9. Cashin A, Potter E, Stevens W, et al. Fit for prison: special population health and fitness programme evaluation. Int $J$ Prison Health 2008; 4(4): 208-216.

10. Perez-Moreno F, Camara-Sanchez M, Tremblay JF, et al. Benefits of exercise training in Spanish prison inmates. Int J Sports Med 2007; 28(12): 1046-1052.

11. Harber MP, Kaminsky LA, Arena R, et al. Impact of cardiorespiratory fitness on all-cause and disease-specific mortality: advances since 2009. Prog Cardiovasc Dis 2017; 60(1): 11-20.

12. Garcia-Hermoso A, Cavero-Redondo I, Ramirez-Velez R, et al. Muscular strength as a predictor of all-cause mortality in an apparently healthy population: a systematic review and meta-analysis of data from approximately 2 million men and women. Arch Phys Med Rehabil 2018; 99(10): 2100-2113.e5.

13. Dhana K, Kavousi M, Ikram MA, et al. Body shape index in comparison with other anthropometric measures in prediction of total and cause-specific mortality. J Epidemiol Community Health 2016; 70(1): 90-96.

14. Boutron I, Altman DG, Moher D, et al. CONSORT statement for randomized trials of nonpharmacologic treatments: a 2017 update and a CONSORT extension for nonpharmacologic trial abstracts. Ann Intern Med 2017; 167(1): 40-47.

15. Slade SC, Dionne CE, Underwood M, et al. Consensus on exercise reporting template (CERT): explanation and elaboration statement. Br J Sports Med 2016; 50: 1428 1437.

16. Rikli $\mathrm{R}$ and Jones C. Development and validation of a functional fitness test for community-residing older adults. J Aging Phys Activ 1999; 7: 127-161.

17. Singh SJ, Morgan MD, Scott S, et al. Development of a shuttle walking test of disability in patients with chronic airways obstruction. Thorax 1992; 47(12): 1019-1024.

18. Peterson MD and Krishnan C. Growth charts for muscular strength capacity with quantile regression. Am J Prev Med 2015; 49(6): 935-938.

19. Gardner DM, Murphy AL, O’Donnell H, et al. International consensus study of antipsychotic dosing. $\mathrm{Am}$ J Psychiatry 2010; 167(6): 686-693.
20. Derogatis L. Brief symptom inventory (BSI)-18: administration, scoring and procedures manual. Minneapolis, MN: NCS Pearson, Inc, 2001.

21. Koehler K and Drenowatz C. Monitoring energy expenditure using a multi-sensor device-applications and limitations of the SenseWear armband in athletic populations. Front Physiol 2017; 8: 983.

22. Hopkins WG, Marshall SW, Batterham AM, et al. Progressive statistics for studies in sports medicine and exercise science. Med Sci Sports Exerc 2009; 41(1): 3-13.

23. Hopkins WG. A spreadsheet to compare means of two groups. Sportsci 2007; 11: 22-24.

24. Battaglia C, di Cagno A, Fiorilli G, et al. Participation in a 9-month selected physical exercise programme enhances psychological well-being in a prison population. Crim Behav Ment Health 2015; 25(5): 343-354.

25. Marzolini S, Jensenc B and Melvillec P. Feasibility and effects of a group-based resistance and aerobic exercise program for individuals with severe schizophrenia: a multidisciplinary approach. Ment Health Phys Act 2009; 2: 29-36.

26. Scheewe TW, Takken T, Kahn RS, et al. Effects of exercise therapy on cardiorespiratory fitness in patients with schizophrenia. Med Sci Sports Exerc 2012; 44(10): 1834 1842.

27. Silva BA, Cassilhas RC, Attux C, et al. A 20-week program of resistance or concurrent exercise improves symptoms of schizophrenia: results of a blind, randomized controlled trial. Braz J Psychiatry 2015; 37(4): 271-279.

28. Pelletier JR, Nguyen M, Bradley K, et al. A study of a structured exercise program with members of an ICCD Certified Clubhouse: program design, benefits, and implications for feasibility. Psychiatr Rehabil J 2005; 29(2): 89-96.

29. Firth J, Carney R, Pownall M, et al. Challenges in implementing an exercise intervention within residential psychiatric care: a mixed methods study. Ment Health Phys Act 2017; 12: 141-146.

30. Hallgren M, Skott M, Ekblom O, et al. Exercise effects on cognitive functioning in young adults with first-episode psychosis: FitForLife. Psychol Med 2019; 49: 431-439.

31. Lavratti C, Dorneles G, Pochmann D, et al. Exerciseinduced modulation of histone $\mathrm{H} 4$ acetylation status and cytokines levels in patients with schizophrenia. Physiol Behav 2017; 168: 84-90.

32. Stubbs B, Vancampfort D, Rosenbaum S, et al. Dropout from exercise randomized controlled trials among people with depression: a meta-analysis and meta regression. $J$ Affect Disord 2016; 190: 457-466.

33. Vancampfort D, Rosenbaum S, Schuch FB, et al. Prevalence and predictors of treatment dropout from physical activity interventions in schizophrenia: a metaanalysis. Gen Hosp Psychiatry 2016; 39: 15-23.

34. Stork MJ, Kwan MY, Gibala MJ, et al. Music enhances performance and perceived enjoyment of sprint interval exercise. Med Sci Sports Exerc 2015; 47(5): 1052-1060. 
35. Munoz-Martinez FA, Rubio-Arias JA, Ramos-Campo DJ, et al. Effectiveness of resistance circuit-based training for maximum oxygen uptake and upper-body one-repetition maximum improvements: a systematic review and metaanalysis. Sports Med 2017; 47(12): 2553-2568.

36. Vancampfort D, Rosenbaum S, Schuch FB, et al. Cardiorespiratory fitness in severe mental illness: a systematic review and meta-analysis. Sports Med 2017; 47(2): 343-352.

37. Davies TB, Kuang K, Orr R, et al. Effect of movement velocity during resistance training on dynamic muscular strength: a systematic review and meta-analysis. Sports Med 2017; 47(8): 1603-1617.

38. Gonzalez-Badillo JJ, Izquierdo $\mathrm{M}$ and Gorostiaga EM. Moderate volume of high relative training intensity produces greater strength gains compared with low and high volumes in competitive weightlifters. $J$ Strength Cond Res 2006; 20(1): 73-81.

39. Firth J, Rosenbaum S, Stubbs B, et al. Motivating factors and barriers towards exercise in severe mental illness: a systematic review and meta-analysis. Psychol Med 2016; 46(14): 2869-2881. 